Draft VERSION OCTOBER 30, 2003

Preprint typeset using $\mathrm{LAT}_{\mathrm{E}} \mathrm{X}$ style emulateapj

\title{
SPECTROSCOPIC DETECTION OF TYPE IA SUPERNOVAE IN THE SLOAN DIGITAL SKY
} SURVEY

\author{
Darren S. Madgwick ${ }^{1,2}$, Paul C. Hewett ${ }^{3}$, Daniel J. Mortlock ${ }^{3}$ \& Lifan Wang ${ }^{1}$ \\ ${ }^{1}$ Lawrence Berkeley National Laboratory, MS50R-5032, Berkeley, CA 94720 \\ ${ }^{2}$ Hubble Fellow \\ ${ }^{3}$ Institute of Astronomy, Madingley Road, Cambridge CB3 0HA, U.K. \\ Draft version October 30, 2003
}

\begin{abstract}
We present the results of a novel new search of the first data-release from the Sloan Digital Sky Survey (SDSS-DR1) for the spectra of supernovae. The use of large spectroscopic galaxy surveys offers the prospect of obtaining improved estimates of the local supernova rate, with the added benefit of a very different selection function to that of conventional photometric surveys. In this Letter we present an overview of our search methodology and the details of 19 Type Ia supernovae found in SDSS-DR1. The supernovae sample is used to make a preliminary estimate, $\Gamma_{\text {Ia }}=0.4 \pm 0.2 h^{2} \mathrm{SNu}$, of the cosmological SNe rate.

Subject headings: methods: data analysis — supernovae: general
\end{abstract}

\section{INTRODUCTION}

Supernovae (SNe) are generally discovered through difference imaging techniques (see e.g. Perlmutter et al. 1995; Schmidt et al. 1998). However, it is also possible to detect them spectroscopically - by identifying the broad peaks and troughs that typically distinguish a SN spectrum from that of its host-galaxy.

The spectroscopic approach to the detection of SNe has a number of advantages; namely that a detection can be made with only one observation, and that the type is readily identifiable without additional follow-up observations. Despite these advantages it is clear that a dedicated spectroscopic SN survey is completely impractical - the rate of detection relative to telescope time is far too low. Fortunately, the data required for such a survey are already available from several large galaxy redshift surveys now approaching completion. In a forthcoming paper, Mortlock, Madgwick \& Hewett 2003 calculate that the $10^{6}$ galaxy spectra to be obtained by the Sloan Digital Sky Survey (SDSS, York et al. 2000) should yield 200 SNe detections.

One immediate application for such a sample is that the cosmological SNe rate, $\Gamma$, can be constrained to $<10$ per cent, a considerable improvement on existing single survey measurements of $\Gamma$ (e.g. Pain et al. 1996, 2002; Hardin et al. 2000). Moreover, using a galaxy redshift survey ensures that the host galaxy sample is extremely well defined, including redshifts, broad-band colours, spectroscopic and morphological types.

The SNe presented in this Letter have typically been observed $\sim 2-3$ years previously, and so follow-up observations are not possible. However, it is important to stress that if a search for SNe is carried out as soon as the spectroscopic data in a given redshift survey is reduced there is potentially a significant amount of time in which more detailed follow-up observations can be carried out.

This Letter describes the first results of an analysis of the $\sim 10^{5}$ galaxy spectra in the SDSS Data Release 1 (SDSS-DR1 Abazajian et al. 2003). The SNe detection procedures are described in $\S 2$, with the efficiency of the technique assessed using simulations in $\S 3$. The actual SNe discoveries are detailed in $\S 4$ before an initial estimate of the cosmological SN rate is presented in $\S 5$. The main results and future possibilities are summarised in $\S 6$.

\section{METHOD}

The spectroscopic detection of SNe relies upon the ability to distinguish the broad spectral features typical of these objects in a composite host-galaxy+SN spectrum where the galaxy spectrum will usually dominate. Two key tasks need to be addressed in order to achieve this goal:

1. The host-galaxy spectrum needs to be 'subtracted' from the observed spectrum, leaving a 'residual' spectrum. The residual spectrum should be dominated either, by noise (if the original spectrum was simply that of a galaxy), or, by SNe spectral features if these are present.

2. The 'residual' spectrum then needs to be searched for the presence of characteristically broad SNe features, which must also be distinguished from any other artifacts that have not been removed in the previous step.

We outline here the steps we have adopted to carry out these tasks.

\subsection{Galaxy subtraction}

Although galaxy spectra appear superficially to exhibit a wide variety of forms, they can be very successfully parameterised with only a handful of components. In particular, the use of a principal component analysis (PCA) has proved to be well adapted to this task (see e.g. Connolly et al. 1995; Folkes, Lahav \& Maddox 1996; Madgwick et al. 2002a).

The key result of the PCA is the construction of an orthogonal set of 'eigenspectra' - each of which successively incorporates the maximum amount of information about the observed galaxy spectral distribution. Previous analyses of samples of galaxy spectra have shown that as few as 8 such eigenspectra can be used to accurately reconstruct most galaxy spectra (Folkes, Lahav \& Maddox 1996). 
In our analysis we perform PCA on a representative sample of 1000 galaxy spectra drawn from the SDSS, to construct a set of 20 eigenspectra. The eigenspectra provide an optimal basis for reconstructing, and then subtracting, each host-galaxy spectrum. The extended wavelength range, excellent resolution and spectrophotometric qualities of the SDSS spectra mean that all 20 components contain information of significance for the spectroscopic reconstructions. However, our technique is not sensitive to the exact number of components employed and the use of only 10 eigenspectra yielded essentially identical results.

The detailed procedure is as follows:

1. The basis of 20 eigenspectra was constructed using PCA on 1000 galaxy spectra, chosen not to contain a strong signature of SNe spectra or any other spectroscopic artifact or anomaly.

2. Each observed spectrum in the SDSS-DR1 was then projected onto this basis. These projections quantify the relative importance of different components in the spectra of the galaxies and the observed spectra can be reconstructed very accurately from the 20 eigenspectra. Unusual spectroscopic features (such as those of $\mathrm{SNe}$ ) do not contribute to the form of the eigenspectra and are therefore not included in the reconstructed galaxy spectra.

3. Each galaxy spectrum was then reconstructed from the eigenspectra. Subtracting the reconstructed spectrum from the original galaxy spectrum produces a 'residual' spectrum. It is this residual spectrum that will be searched for the SNe features.

\subsection{SNe matching}

The residual spectrum left after subtracting the reconstructed host-galaxy spectrum is almost always dominated by noise, validating the effectiveness of the PCAreconstructions. However, spectroscopic artifacts were present for a small fraction of the objects due to poor sky subtraction or residual nebular emission lines, the latter resulting from particularly unusual emission line ratios. To reduce the impact of these relatively high-frequency residuals, a low-pass filtering of the residual spectra was applied before attempting to identify SN features. The filtering was based upon the á trous wavelet transform (Starck, Siebenmorgen \& Gredel 1997 and references therein), as described in Madgwick et al. 2002b. This additional filtering leaves a low-pass filtered residual spectrum, $f_{\lambda}$, allowing us to isolate just the broadest spectral features without contamination from narrow peaks and troughs present in the original observed spectrum.

In order to quantify the significance of a SN detection we use two parameters - the correlation coefficient (here denoted by CC) and the RMS signal. The first of these parameters, CC, is determined simply by calculating the covariance between each low-pass filtered residual spectrum, $f_{i}\left(i=0, . . N_{\text {gal }}\right)$ and a sequence of different SN templates, $t_{j}\left(j=0, . . N_{\text {temp }}\right)^{1}$,

\footnotetext{
${ }^{1}$ We make use of the observed spectra from SN1998aq at $-9,0$, 7, 19, 31 and 55 days (Branch et al. 2003), SN1998bu at $-4,8,13$, 28 days (Jha et al. 1999) and SN1999ee at $-8,-1,12,20,33$ days (Hamuy et al. 2002), as these cover all available spectral features uniformly over a wide range of times.
}

$$
\mathrm{CC}_{\mathrm{ij}}=\frac{\operatorname{Cov}_{\mathrm{f}_{\mathrm{i}} \mathrm{t}_{\mathrm{j}}}}{\sigma_{\mathrm{i}} \sigma_{\mathrm{j}}} .
$$

For each galaxy $i$, we set $\mathrm{C}_{i}=\max \left(\mathrm{C}_{\mathrm{ij}}\right)$, so that only the 'best-fitting' SN template is used to determine the likelihood of a given detection. The resulting value of CC quantifies the similarity between the shape of the residual spectrum and the shape of the best-fitting SN template.

The RMS signal is also calculated from the low-pass filtered residual spectrum. This parameter provides a measure of the amplitude of the signal present in the residual spectrum.

$$
\operatorname{RMS}=\sqrt{\left(\frac{\sum_{\lambda} f_{\lambda}}{N_{\lambda}}\right)^{2}} .
$$

\section{SIMULATIONS}

There are several criteria which determine whether a $\mathrm{SN}$ will be detectable in a given spectrum. One of the most important of these is the relative brightness of the SN compared with the host-galaxy - which will determine the prominence of the SN features in the observed composite host-galaxy+SN spectrum.

In order to test how important these effects are we simulated several thousand composite 'host-galaxy+SN' spectra, by randomly choosing objects from the SDSSDR1 and adding our SNe templates. The flux in the galaxy spectra and SN templates was calculated over the wavelength range $5000-6000 \AA$, i.e. approximating the $V$-band, and SNe templates were added with flux ratios (SN/galaxy) in the range 0.75-0.1. For each simulated spectrum we then ran our detection algorithm and calculated both the CC and RMS values. It was determined from these simulations that our algorithms work very well at identifying a SN component in the spectra if the SN contributes $\geq 10 \%$ of the $V$-band flux recorded in the SDSS fibre-spectrum (more details will be presented in Mortlock, Madgwick \& Hewett 2003).

An empirical cut in $\mathrm{CC}$ and RMS allows the detection of virtually all $\mathrm{SNe}$ present down to a relative flux ratio of 0.1 (corresponding to a magnitude difference $\Delta m \simeq 2$ between the host-galaxy and SN).

\section{RESULTS}

We have calculated the CC and RMS parameters for all 115,977 objects in SDSS-DR1 with $z \leq 0.25, z_{\text {confidence }} \geq$ 0.35 and a spectroscopic classification of 'galaxy'.

Some 1500 SDSS-DR1 spectra satisfied our selection criteria, representing $\sim 1 \%$ of this spectroscopic galaxy sample. However, although we inspected visually all of these spectra, high-confidence detections of SN were only made for objects which had $\mathrm{CC} \geq 0.6$ - corresponding to only the 230 most significant detections. The detection algorithm has proved highly successful in identifying spectra with SNe present at high confidence.

In total, 19 Type Ia SNe were identified with high confidence from our search. Another 15 possible detections were made, although further work is required both to quantify the true fraction of $\mathrm{SNe}$ in this sub-sample and to optimise the detection efficiency for the presence of $\mathrm{SNe}$ at low flux ratios. Properties of the high-confidence $\mathrm{SNe}$ detections are given in Table 1. 
TABLE 1

Type Ia SNe discovered in SDSS-DR1. In each Case the SNe type, magnitude and Ages have been Derived using a Wide Variety of SNe templates (InCluding 35 Type Ib/C and 41 Type Ia spectra). The Quoted ages are the $\pm 1 \sigma$ Range about the expected value. Also given are the flux ratios between the galaxy and SN components of EACH FIBRE-SPECTRUM - AS DERIVED FROM THE SAME TEMPLATE LIBRARY.

\begin{tabular}{|c|c|c|c|c|c|c|c|}
\hline Name & R.A. & Dec & $\overline{V_{\mathrm{SN}} \mathrm{mag}}$ & Flux ratio & $\mathrm{Z}$ & Date observed & SN age (days) \\
\hline $2000 \mathrm{fz}$ & $01 \quad 18 \quad 35.83$ & +144100.5 & 17.8 & 0.40 & 0.054 & $2000-12-15$ & $6.3-6.8$ \\
\hline $2000 \mathrm{ga}$ & 022903.73 & -082413.7 & 20.0 & 0.14 & 0.141 & $2000-12-24$ & $6.0-7.9$ \\
\hline $2001 \mathrm{kl}$ & 030851.44 & -011024.1 & 20.4 & 0.06 & 0.126 & $2001-01-22$ & $\sim 20$ \\
\hline $2001 \mathrm{ks}$ & 075646.50 & +365917.0 & 18.7 & 0.12 & 0.078 & 2001-04-18 & $-5.0--3.0$ \\
\hline 2000fy & 080312.61 & +473649.7 & 19.5 & 0.19 & 0.117 & $2000-12-06$ & $2.5-3.5$ \\
\hline $2001 \mathrm{kn}$ & 083209.85 & +471727.6 & 20.0 & 0.17 & 0.134 & 2001-03-13 & $-5.0-3.0$ \\
\hline $2001 \mathrm{~km}$ & 091138.38 & -004254.0 & 18.4 & 0.56 & 0.070 & 2001-02-15 & $6.0-6.3$ \\
\hline $2001 \mathrm{kj}$ & 092229.15 & +575429.3 & 18.1 & 0.30 & 0.063 & 2001-01-02 & $10-11$ \\
\hline $2001 \mathrm{kq}$ & 093334.31 & +551026.0 & 18.5 & 0.08 & 0.074 & 2001-03-23 & $-4.9--3.1$ \\
\hline $2001 \mathrm{kp}$ & 095153.08 & +010605.8 & 18.2 & 0.34 & 0.063 & 2001-03-21 & $-3.0--2.0$ \\
\hline $2001 \mathrm{kr}$ & 095915.75 & +005802.4 & 19.1 & 0.43 & 0.088 & $2001-03-26$ & $11-12$ \\
\hline $2000 f x$ & 101800.47 & -000158.0 & 18.7 & 0.26 & 0.065 & $2000-12-01$ & $16-18$ \\
\hline 2001ki & 104858.40 & -002645.2 & 19.3 & 0.14 & 0.106 & 2001-01-01 & $6.0-7.3$ \\
\hline $2001 \mathrm{kk}$ & 124733.41 & +000557.1 & 18.8 & 0.15 & 0.086 & 2001-01-19 & $5.7-7.8$ \\
\hline $2001 \mathrm{ku}$ & 133944.77 & +622337.0 & 19.9 & 0.15 & 0.136 & 2001-06-19 & $3.3-4.0$ \\
\hline $2001 \mathrm{ko}$ & 141058.32 & +645050.9 & 20.0 & 0.15 & 0.141 & 2001-03-16 & $7.6-8.4$ \\
\hline $2000 \mathrm{fw}$ & 143014.07 & +003035.4 & 19.1 & 0.28 & 0.096 & 2000-03-10 & $-4.6--3.3$ \\
\hline $2001 \mathrm{kt}$ & $1617 \quad 13.40$ & +482827.7 & 19.6 & 0.30 & 0.104 & $2001-05-25$ & $14-17$ \\
\hline $2000 \mathrm{gb}$ & 173228.54 & +560425.5 & 20.4 & 0.22 & 0.123 & $2000-10-01$ & $16-20$ \\
\hline
\end{tabular}
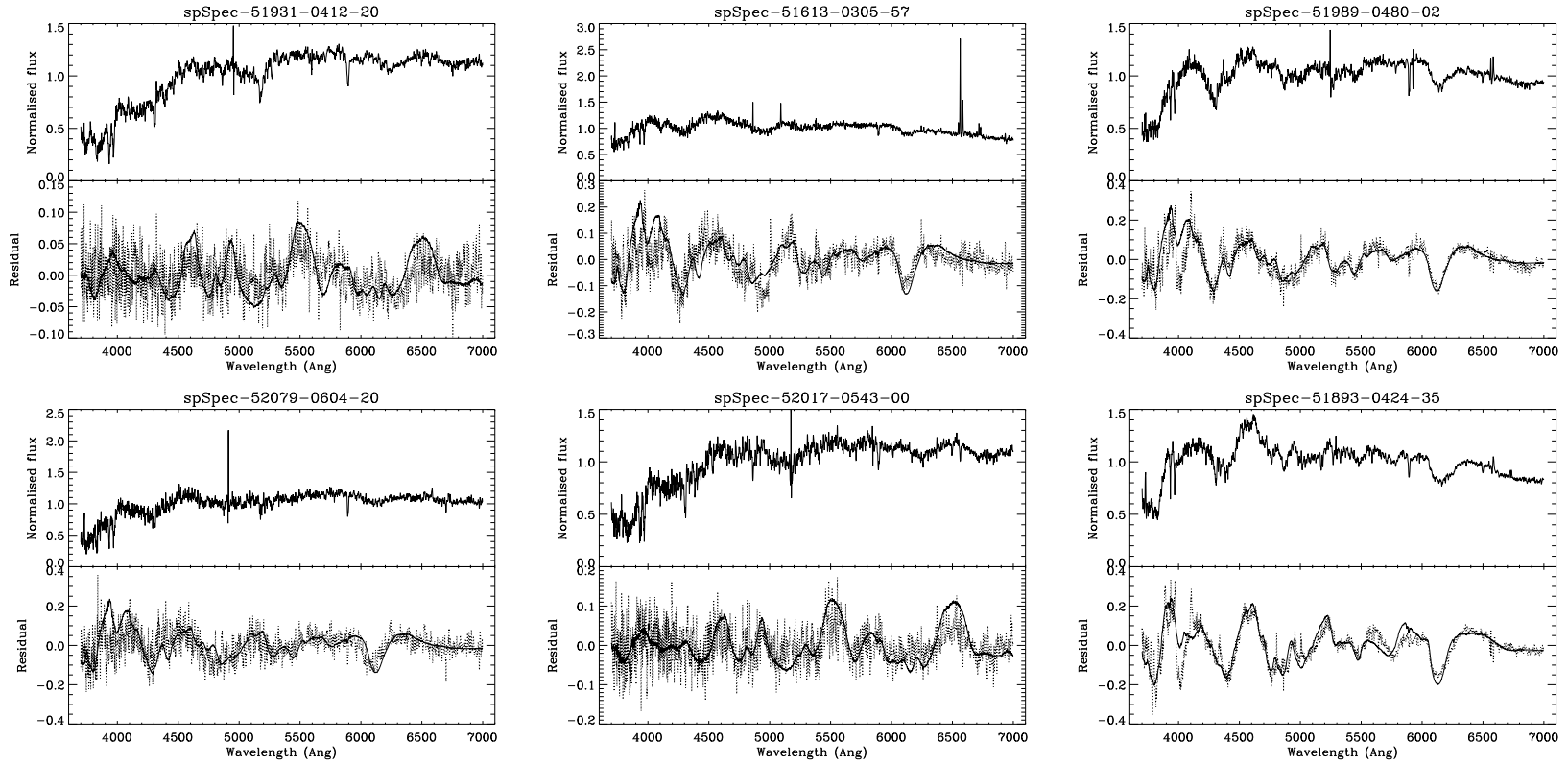

FIG. 1.- Example spectra of the SNe identified in SDSS-DR1 are shown in each panel. In addition, the lower plot in each panel shows the 'residual' spectrum that is left after subtracting the host-galaxy from the observed spectrum. The best-fitting template SN spectrum is shown overplotted on this residual spectrum. 


\section{THE COSMOLOGICAL SNE RATE}

A crude estimate of the number of SNe expected in the survey, $\left\langle N_{\mathrm{SN}}\right\rangle$, is given by the product of four quantities: the number of galaxies in the survey, $N_{\mathrm{g}}$; the average luminosity of a survey galaxy, $\left\langle L_{\mathrm{g}}\right\rangle$; the typical control time for which a $\mathrm{SN}$ is sufficiently bright to be detectable, $\left\langle t_{\mathrm{SN}}\right\rangle$; and, of course, the SN rate itself (which is conventionally measured in supernova units, $\mathrm{SNu}^{2}$ ). Taking typical values for these quantities yields

$$
\left\langle N_{\mathrm{SN}}\right\rangle \simeq 20 \frac{N_{\mathrm{g}}}{10^{5}} \frac{\left\langle L_{\mathrm{g}}\right\rangle}{L_{*}} \frac{\left\langle t_{\mathrm{SN}}\right\rangle}{10 \text { days }} \frac{\Gamma}{1 \mathrm{SNu}},
$$

where $L_{*} \simeq 10^{10} L_{\odot}($ Madgwick et al. 2002a) is the fiducial $B$-band luminosity of a field galaxy. The most difficult parameter to estimate is $\left\langle t_{\mathrm{SN}}\right\rangle$, which is determined from a combination of the SNe light-curve shapes, the positional distribution of the SNe in their host-galaxies, the size of the apertures used to obtain the spectra and the detection algorithm used. A value of $\left\langle t_{\mathrm{SN}}\right\rangle \simeq 20$ days is consistent both with the best fit ages of the SNe listed in Table 1 and the more theoretical approach taken by Mortlock, Madgwick \& Hewett 2003, but is probably uncertain by up to $50 \%$ in this preliminary analysis.

The estimate of the SN rate from the discovery of 19 type Ia SNe in the SDSS-DR1 galaxy spectra is thus

$$
\Gamma_{\text {Ia }}=0.4 \pm 0.2 h^{2} \mathrm{SNu}
$$

where both the Poisson uncertainty in the sample size and our estimated uncertainty in the control time, $\left\langle t_{\mathrm{SN}}\right\rangle$, are included. $h$ is the value of the Hubble Constant in units of $100 \mathrm{kms}^{-1} \mathrm{Mpc}^{-1}$. Our estimate is consistent with previous measurements at high redshifts: $\Gamma_{\mathrm{Ia}}(z=0.4)=0.8 \pm$ $0.5 h^{2} \mathrm{SNu}$ (Pain et al. 1996), $\Gamma_{\mathrm{Ia}}(z=0.5) \simeq 0.58 \pm 0.2 h^{2}$ $\mathrm{SNu}$ (Pain et al. 2002), and is very similar to that previously determined at low- $z: \Gamma_{\mathrm{Ia}}(z=0.1)=0.4 \pm 0.3 h^{2} \mathrm{SNu}$ (Hardin et al. 2000).

\section{CONCLUSIONS}

A novel spectroscopic analysis of the $\sim 10^{5}$ galaxy spectra in the SDSS-DR1 has resulted in the definite identification of 19 Type Ia SNe, along with a similar number of less certain detections. The resultant estimate of the local Type Ia $\mathrm{SN}$ rate, $\Gamma_{\mathrm{Ia}} \simeq 0.4 \pm 0.2 h^{2} \mathrm{SNu}$, is consistent with that obtained by more conventional imaging methods, although the uncertainties in all cases are dominated by the small number of SNe.

The future for spectroscopic SNe searches is promising, as this methodology can be applied to any redshift survey for which a large sample of galaxy spectra exist, opening up new scientific applications for present and future surveys. A more complete analysis of the SDSS-DR1 spectra will include a more sophisticated estimate of the Cosmological SNe rate (Mortlock, Madgwick \& Hewett 2003), along with a number of consistency checks in which the magnitudes, redshifts and epochs (relative to maximum light) of the detected SNe are compared to the predicted distributions.

Looking ahead, the ease with which these Type Ia SNe have been discovered in the SDSS-DR1 spectra implies

\footnotetext{
$21 \mathrm{SNu}$ is defined as the number of SNe per century per $10^{10}$ Solar luminosities in the $B$-band (i.e. $1 \mathrm{SNu}=10^{-12} \mathrm{yr}^{-1} L \odot^{-1}$ )
}

that the full survey of $\sim 10^{6}$ galaxies should yield at least $\sim 200 \mathrm{SNe}$ of all types. Such a sample should provide an estimate of $\Gamma$ which is largely free of systematic errors and subject to only a small statistical uncertainty. Furthermore, the spectroscopic selection applied to the SDSS sample will allow the frequency of SNe events in terms of the properties of the underlying galaxy population to be determined to unprecedented accuracy (see e.g. Cappellaro et al. 1997; Cappellaro, Evans \& Turatto 1997).

A final important point to make is that all of these SNe have been discovered several years after their occurrence, and as such follow-up observations are simply not possible. However, by incorporating our search methodology into the SDSS (and other survey's) data reduction pipelines these objects can be discovered essentially immediately. It is hoped that the presentation of the results in this Letter will result in this occurring, so that this interesting aspect of galaxy redshift surveys no longer goes neglected.

DSM would like to thank Saul Perlmutter, Alex Kim and Eric Linder for many useful discussions. Support for this work was provided by NASA through Hubble Fellowship grant \#HST-HF-01163.01-A awarded by the Space Telescope Science Institute, which is operated by the Association of Universities for Research in Astronomy, Inc., for NASA, under contract NAS 5-26555. DJM was supported by PPARC.

\section{REFERENCES}

Abazijian K., et al., (the SDSS Collaboration), 2003, ApJ, submitted [astro-ph/0305492]

Branch D., et al., 2003, AJ, 126, 1489

Cappellaro E., et al., 1997, A\&A, 322, 431

Cappellaro E., Evans R., Turatto M., 1999, A\&A, 351, 459

Connolly A. J., Szalay A. S., Bershady M. A., Kinney A. L., Calzetti D., 1995, AJ, 110, 1071

Folkes S., Lahav O., Maddox S., 1996, MNRAS, 283, 651

Jha S., et al., 1999, ApJS, 125, 73

Hamuy M., et al., 2002, AJ, 124, 417

Hardin, D., et al., 2000, A\&A, 362, 419

Madgwick, D. S., et al., (the 2dFGRS Team), 2002a, MNRAS, 333, 133

Madgwick D. S., Hewett P. C., Mortlock D. J., Lahav O., 2002b, MNRAS, 334, 209

Mortlock, D. J., Madgwick, D. S, \& Hewett, P. C., 2003, MNRAS, in preparation

Pain, R., et al., 1996, ApJ, 473, 356

Pain, R., et al., 2002, ApJ, 577, 120

Perlmutter, S., et al., 1995, ApJ, 440, 41

Riess, A. G., et al., 1997, AJ, 114, 722

Schmidt, B. P., et al., 1998, ApJ, 507, 46

Starck, J., Siebenmorgen R., Gredel R., 1997, ApJ, 482, 1011

York, D. G., et al., 2000, AJ, 120, 1579 